\title{
Sympathetic $\beta_{1}$-adrenergic signaling contributes to regulation of human bone metabolism
}

\author{
Sundeep Khosla, ${ }^{1}$ Matthew T. Drake, ${ }^{1}$ Tammie L. Volkman, ${ }^{1}$ Brianne S. Thicke, ${ }^{1}$ Sara J. Achenbach, ${ }^{2}$ Elizabeth J. Atkinson, ${ }^{2}$ \\ Michael J. Joyner, ${ }^{3}$ Clifford J. Rosen, ${ }^{4}$ David G. Monroe, ${ }^{1}$ and Joshua N. Farr ${ }^{1}$ \\ ${ }^{1}$ Robert and Arlene Kogod Center on Aging and Division of Endocrinology, ${ }^{2}$ Department of Health Sciences Research, and ${ }^{3}$ Department of Anesthesia, Mayo Clinic College of Medicine and Science, Rochester, \\ Minnesota, USA. ${ }^{4}$ Maine Medical Center Research Institute, Scarborough, Maine, USA.
}

\begin{abstract}
BACKGROUND. Evidence from rodent studies indicates that the sympathetic nervous system (SNS) regulates bone metabolism, principally via $\boldsymbol{\beta}_{2}$-adrenergic receptors ( $\left.\beta_{2}-A R s\right)$. Given the conflicting human data, we used multiple approaches to evaluate the role of the SNS in regulating human bone metabolism.
\end{abstract}

METHODS. Bone biopsies were obtained from 19 young and 19 elderly women for assessment of ADRB1, ADRB2, and ADRB3 mRNA expression. We examined the relationship of $\beta$-blocker use to bone microarchitecture by high-resolution peripheral quantitative CT in a population sample of 248 subjects. A total of 155 postmenopausal women were randomized to 1 of 5 treatment groups for $\mathbf{2 0}$ weeks: placebo; propranolol, 20 mg b.i.d.; propranolol, $\mathbf{4 0}$ mg b.i.d.; atenolol, 50 mg/day; or nebivolol, $5 \mathrm{mg} /$ day. We took advantage of the $\beta_{1}$-AR selectivity gradient of these drugs (propranolol [nonselective] $<<$ atenolol [relatively $\beta_{1}$-AR selective] < nebivolol [highly $\beta_{1}$-AR selective]) to define the $\beta$-AR selectivity for SNS effects on bone.

RESULTS. ADRB1 and ADRB2, but not ADRB3, were expressed in human bone; patients treated clinically with $\beta_{1}$-AR-selective blockers had better bone microarchitecture than did nonusers, and relative to placebo, atenolol and nebivolol, but not propranolol, reduced the bone resorption marker serum C-telopeptide of type I collagen (by $19.5 \%$ and $20.6 \%$, respectively; $P<0.01$ ) and increased bone mineral density of the ultradistal radius (by $3.6 \%$ and $2.9 \% ; P<0.01$ and $P<0.05$, respectively).

CONCLUSIONS. These 3 independent lines of evidence strongly support a role for adrenergic signaling in the regulation of bone metabolism in humans, principally via $\beta_{1}$-ARs.

TRIAL REGISTRATION. ClinicalTrials.gov NCT02467400.

FUNDING. This research was supported by the NIH (AG004875 and AR027065) and a Mayo Clinic Clinical and Translational Science Award (CTSA) (UL1 TR002377).

\section{Introduction}

There is considerable evidence from animal studies that the sympathetic nervous system (SNS) is an important regulator of bone metabolism. The Karsenty group showed that intracerebroventricular administration of leptin reduced bone formation and bone mass in mice (1). This effect was mediated via the SNS, with leptin acting on the hypothalamus to increase sympathetic outflow, leading to the activation of $\beta$-adrenergic receptors ( $\beta$-ARs) on osteoblasts and resulting in decreases in osteoblast proliferation, differentiation, and histologically assessed bone formation (2). Further, SNS activation also resulted in greater osteoclastic bone resorption

\section{Related Commentary: p. 4745}

Role of funding source: These studies were funded by the NIH, which did not play a role in the performance of the studies.

Conflict of interest: The authors have declared that no conflict of interest exists. License: Copyright 2018, American Society for Clinical Investigation.

Submitted: May 10, 2018; Accepted: August 23, 2018.

Reference information: J Clin Invest. 2018;128(11):4832-4842.

https://doi.org/10.1172/JCI122151 by increasing osteoblast expression of RANKL (3). Subsequent studies from the same group using osteoblast-specific deletion of $\beta_{2}$-ARs indicated that the effects of sympathetic outflow on bone, at least in mice, were mediated principally via $\beta_{2}$-ARs, with little or no role for $\beta_{1}$ - or $\beta_{3}$-ARs (4). However, other investigators have identified both $\beta_{1}$ - and $\beta_{3}$-ARs in various osteoblast-like cell lines (5), and global KO in mice of either $\beta_{1}$ - or $\beta_{2}$-ARs was associated with skeletal changes (6), indicating that both receptor subtypes may play important roles in regulating bone metabolism.

On the basis of these findings, several animal studies have examined the possible effects of pharmacological $\beta$-AR blockade on bone mass and metabolism. Takeda et al. (2) demonstrated that 5 weeks of treatment with the nonselective $\beta$-blocker propranolol $(0.4 \mathrm{mg} /$ day $)$, increased bone mass in ovary-intact female mice. Extending their findings to mice ovariectomized at 6 weeks of age and treated for 7 weeks, these investigators found that the same propranolol dose maintained bone mass at levels comparable to those in sham-operated animals. These results were subsequently confirmed in 2 studies in rats, both of which also found important dose-related effects of propranolol on bone metabolism, with lower doses being more effective than higher ones $(7,8)$. In the 
Table 1. Expression of ADRB1, ADRB2, and ADRB3 mRNA levels in human bone biopsies and cultured osteoblasts assessed by RNA-seq

\begin{tabular}{|c|c|c|c|c|c|}
\hline \multirow[b]{2}{*}{ Human biopsies } & \multicolumn{2}{|c|}{ Raw counts, median (range) } & \multicolumn{3}{|c|}{ RPKM, median (range) } \\
\hline & Young $(n=19)$ & Elderly $(n=19)$ & Young ( $n=19$ ) & Elderly $(n=19)$ & $P$ value \\
\hline ADRB1 & $62(44,106)$ & $78(28,106)$ & $0.28(0.17,0.55)$ & $0.36(0.14,0.60)$ & 0.63 \\
\hline ADRB2 & $926(606,1234)$ & $920(564,1340)$ & $5.57(3.29,7.01)$ & $6.16(3.77,8.71)$ & 0.28 \\
\hline$A D R B 3$ & $0(0,2)$ & $0(0,4)$ & $0(0,0.01)$ & $0(0,0.02)$ & 0.95 \\
\hline \multicolumn{6}{|l|}{ hFOB cells $(n=3)$} \\
\hline ADRB1 & \multicolumn{2}{|c|}{$140,150,168$} & \multicolumn{3}{|c|}{$0.29,0.37,0.37$} \\
\hline ADRB2 & \multicolumn{2}{|c|}{$1070,1358,1476$} & \multicolumn{3}{|c|}{$3.44,3.69,4.20$} \\
\hline ADRB3 & \multicolumn{2}{|c|}{$0,0,4$} & \multicolumn{3}{|c|}{$0.00,0.00,0.01$} \\
\hline
\end{tabular}

The $P$ value compares elderly versus young biopsies using a negative binomial regression model. RPKM, reads per kb per million mapped reads.

expression being higher than ADRB1 expression. We found no effect of age on mRNA expression of any of the $\beta$-ARs. ADRB3 mRNA was also not expressed in a human osteoblast cell line (hFOB) (11); the absolute and relative expression levels of $A D R B 1$ and $A D R B 2$ in hFOB cells were very similar to those observed in the human bone biopsies. To establish the functionality of $\beta$-ARs in human osteoblasts, we next treated hFOB cells with either a $\beta_{1}$-AR agonist (dobutamine) $(12,13)$ or a $\beta_{2}$-AR agonist (salmeterol) (14). Both dobutamine and salmeterol increased the mRNA levels of previously identified $\beta$-adrenergic target genes, including C-FOS and C-MYC (Supple-

first, Bonnet et al. (7) ovariectomized or sham operated 6-monthold Wistar rats and treated the ovariectomized rats with $0.1,5$, or $20 \mathrm{mg} / \mathrm{kg}$ propranolol (5 days/week) for 10 weeks. Bone mass and microarchitecture were preserved in the $0.1-\mathrm{mg} / \mathrm{kg}$ group, and this was accompanied by increased osteoblast activity and reduced osteoclast surface, whereas the $20-\mathrm{mg} / \mathrm{kg}$ dose was completely ineffective. These findings were confirmed by Sato et al. (8), who showed in spontaneously hypertensive rats that propranolol doses of 0.1 and $1 \mathrm{mg} / \mathrm{kg} /$ day increased bone mass and indices of bone formation, while decreasing bone resorption indices. By contrast, a dose of $10 \mathrm{mg} / \mathrm{kg} /$ day was less effective. The reason(s) why lower doses of propranolol were more effective than higher doses in regulating bone metabolism in rodents is unclear but is an important issue to consider when interpreting human studies on SNS regulation of bone metabolism. Specifically, a prospective study by Reid et al. (9) examining possible effects of a $\beta$-blocker on bone turnover used a very high dose of propranolol (160 mg/ day) and failed to find any clear effects of propranolol on serum markers of bone turnover. Thus, given the animal dose-response data, the question of whether lower doses of propranolol may have more favorable effects on bone turnover in humans, as well as the $\beta$-AR selectivity ( $\beta_{1}$ - vs. $\beta 2$ - vs. $\beta_{3}$-ARs) for SNS effects on bone in humans remain important, unresolved issues.

In the present study, we used multiple approaches to evaluate the possible role of sympathetic outflow in regulating bone metabolism in humans: (a) examination of the expression of $A D R B 1$, $A D R B 2$, and $A D R B 3$ in human bone biopsies and cultured human bone cells; (b) analysis of population data on bone microarchitecture in subjects either clinically treated or not with $\beta$-blockers; and (c) a direct interventional study that also sought to define the $\beta$-AR selectivity for SNS effects on human bone.

\section{Results}

Expression of $\beta$-ARs in human bone and cultured osteoblasts. We have previously obtained needle biopsies of bone from the posterior iliac crest of 19 young women (mean age \pm SD, $30.3 \pm 5.4$ years) and 19 elderly women (73.1 \pm 6.6 years of age) (10). Table 1 shows the RNA-sequencing (RNA-seq) analysis for $A D R B 1, A D R B 2$, and $A D R B 3$ in these subjects. Both $A D R B 1$ and $A D R B 2$, but not $A D R B 3$, were expressed in human bone samples, with $A D R B 2$ mRNA mental Figure 1, A and B; supplemental material available online with this article; https://doi.org/10.1172/JCI122151DS1), and altered the mRNA levels of a number of $\beta$-adrenergic-responsive circadian clock genes (Supplemental Figure 1, C-F) $(15,16)$. In addition, both dobutamine and salmeterol increased RANKL mRNA levels by approximately 2-fold (Figure 1A), but had no effect on $O P G$ mRNA levels (Figure 1B). Both $\beta$-AR agonists also increased $A D R B 1$ mRNA levels by approximately 4 - to 6-fold (Figure 1C), but neither agonist altered $A D R B 2 \mathrm{mRNA}$ levels (Figure 1D).

Relationship of $\beta$-blocker use to bone microarchitecture in a population-based sample. From our previously described population-based cohort $(17,18)$, we identified 67 subjects over the age of 50 years who had used $\beta$-blockers for at least 1 year over the preceding 5 years; of these, 63 were on $\beta_{1}$-selective blockers (atenolol or metoprolol). Given the insufficient number of subjects on $\beta$-nonselective blockers (propranolol; $n=4$ ), we focused our analysis on those taking $\beta_{1}$-selective blockers. Table 2 shows the demographic characteristics and high-resolution peripheral quantitative CT (HR-pQCT) imaging data for the $\beta_{1}$-selective blocker users and nonusers, adjusted for age and sex. As is evident in Table 2, trabecular microarchitectural parameters (trabecular bone volume as a function of tissue volume [BV/TV] and trabecular number [TbN]) assessed by HR-pQCT at both the radius and tibia were significantly better in the $\beta_{1}$-selective blocker users as compared with nonusers, whereas the cortical parameters were not significantly different. Supplemental Table 1 shows the dual-energy x-ray absorptiometry (DXA) and central (spine and femur neck) QCT parameters, which were not significantly different between the 2 groups.

Interventional study of effects of $\beta$-blockers on bone turnover and bone mineral density. Having demonstrated the expression of $A D R B 1$ and $A D R B 2$, but not $A D R B 3$, in human bone and cultured osteoblasts, as well as better bone microarchitecture in a population sample of $\beta_{1}$-selective blocker users as compared with nonusers, we next sought to definitively establish causality in a proof-of-concept interventional study. To do so, we recruited 165 postmenopausal women and randomized them to 1 of 5 treatment groups for 20 weeks (see Figure 2 for the study CONSORT flow diagram): (a) placebo; (b) propranolol, $20 \mathrm{mg}$ b.i.d.; (c) propranolol, 40 mg b.i.d.; (d) atenolol, $50 \mathrm{mg}$ /day; or (e) nebivolol, $5 \mathrm{mg} /$ day. A total of 155 women received the allocated intervention and 
A
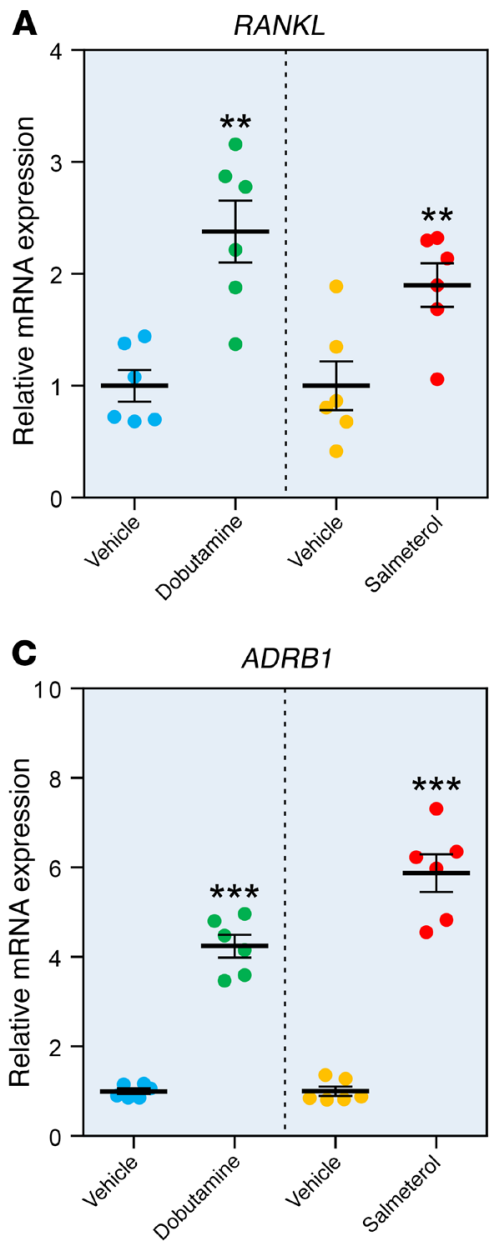

B

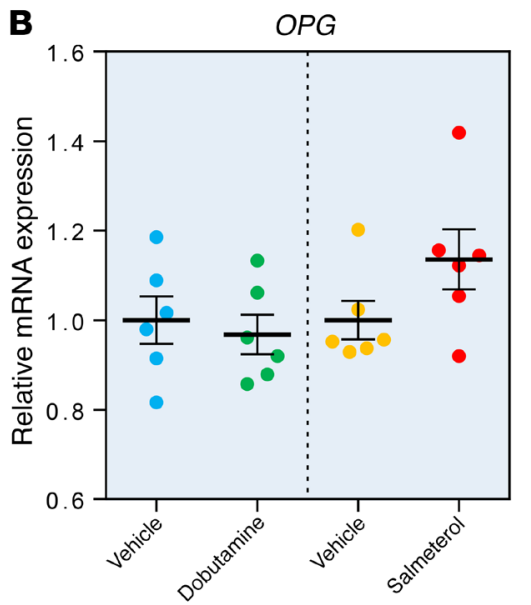

D

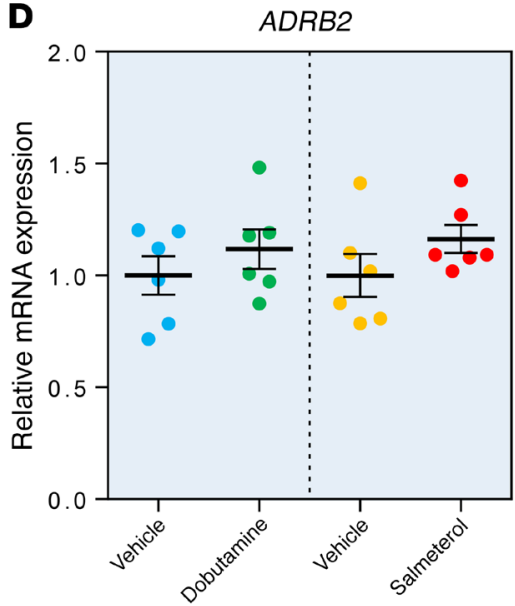

Figure 1. Effects of a $\beta_{1}-A R$ agonist (dobutamine) and a $\beta_{2}$-AR agonist (salmeterol) on gene expression in hFOB cells. Relative mRNA levels of (A) RANKL (also known as TNFSF11), (B) OPC (also known as TNFRSF11B), (C) ADRB1, and (D) ADRB2 in hFOB cells treated for 2 hours with vehicle, dobutamine ( $\beta_{1}-$ AR agonist), or salmeterol $\left(\beta_{2}-A R\right.$ agonist). See Methods for experimental details. $n=6$ per treatment, with comparisons between vehicle and treatment groups using a 2-sample $t$ test. ${ }^{*} P<0.01$ and ${ }^{* *} P<0.001$.
129 completed the full 20 weeks of the study. The propranolol doses were chosen on the basis of a previous human study that had used $160 \mathrm{mg} /$ day and found no significant effects on bone turnover (9) and on the basis of animal data showing, paradoxically, that lower doses of propranolol had greater skeletal efficacy than did higher doses $(7,8)$; as such, we chose to use $50 \%$ and $25 \%$ of the previously ineffective $160-\mathrm{mg}$ /day dose (9). Moreover, as no clinically available $\beta_{2}$-AR-selective antagonists exist, we took advantage of the $\beta_{1}$-AR selectivity gradient of these drugs (propranolol [nonselective] $<<$ atenolol [relatively $\beta_{1}$-AR selective/some $\beta_{2}$-AR antagonism] $<$ nebivolol [highly $\beta_{1}$-AR selective] $)(19,20)$ to define the $\beta$-AR selectivity for SNS effects on bone metabolism.

Table 3 shows the baseline anthropometric, biochemical, and DXA bone mineral density (BMD) data for the study participants. Prespecified primary endpoints were the changes in the bone resorption marker serum C-telopeptide of type I collagen (CTx) and the bone formation marker amino-terminal propeptide of type I collagen (PINP) from baseline out to 20 weeks in each group (Figure 3, A and B). Relative to the placebo group, there were small, nonsignificant decreases in serum CTx levels with both doses of propranolol. By contrast, serum CTx levels decreased significantly following treatment with atenolol (19.5\% relative to placebo) and nebivolol (20.6\% relative to placebo) (Figure 3A). Likewise, serum PINP levels did not change significantly relative to those for placebo with either dose of propranolol, but these levels decreased significantly following treatment with atenolol and nebivolol (Figure
3B). Prespecified secondary endpoints included the changes in the bone resorption marker serum tartrate-resistant acid phosphatase $5 \mathrm{~b}$ (TRAP5b) and the bone formation marker osteocalcin from baseline out to 20 weeks in each group (Figures 3, C and D). As with CTx, serum TRAP5b levels were reduced following treatment with atenolol and nebivolol (by $13.6 \%$ and $15.0 \%$, respectively, relative to placebo; Figure 3C). Propranolol at $20 \mathrm{mg}$ b.i.d. significantly reduced serum TRAP5b levels, but the change observed with the 40 -mg b.i.d. dose was not significant $(P=0.066)$. The changes in serum osteocalcin levels are shown in Figure 3D. Relative to placebo, the small reduction in osteocalcin following atenolol or nebivolol treatment was not statistically significant $(P=0.11$ and $P=$ 0.054 , respectively). By contrast, both doses of propranolol markedly decreased serum osteocalcin levels. Supplemental Figures 2-5 show the time course of changes in the serum bone turnover markers, which were generally similar for atenolol and nebivolol, but differed from the changes seen with the 2 doses of propranolol. Thus, both atenolol and nebivolol treatment resulted in progressive decreases in serum CTx levels over time (Supplemental Figure 2). We observed a transient (week 2) increase in serum PINP levels following nebivolol treatment, but then a subsequent reduction in PINP with both atenolol and nebivolol (Supplemental Figure 3). Changes in serum TRAP5b levels across groups were similar to those seen for CTx (Supplemental Figure 4). As noted earlier, there were minimal changes over time in serum osteocalcin with atenolol or nebivolol treatment, but marked reductions in serum 
Table 2. Demographic and HR-pQCT imaging data on $\beta_{1}$-AR-selective blocker users versus nonusers

\begin{tabular}{|c|c|c|c|}
\hline & Nonusers & Users & $\begin{array}{l}P \text { value (adjusted } \\
\text { for sex and age) }\end{array}$ \\
\hline$n$ & 185 & 63 & - \\
\hline Male, $n(\%)^{A}$ & $94(51 \%)$ & $41(65 \%)$ & - \\
\hline Age, $y r^{A}$ & $66 \pm 10$ & $74 \pm 12$ & - \\
\hline Height, cm & $169 \pm 0.5$ & $169 \pm 0.8$ & 0.39 \\
\hline Weight, kg & $85 \pm 1.3$ & $84 \pm 2.3$ & 0.91 \\
\hline $\mathrm{BMI}, \mathrm{kg} / \mathrm{m}^{2}$ & $29 \pm 0.4$ & $30 \pm 0.7$ & 0.80 \\
\hline \multicolumn{4}{|l|}{ HR-pQCT, trabecular } \\
\hline \multicolumn{4}{|l|}{ Radius } \\
\hline $\mathrm{BV} / \mathrm{TV}$ & $0.130 \pm 0.002$ & $0.142 \pm 0.004$ & 0.023 \\
\hline $\mathrm{TbN}, 1 / \mathrm{mm}$ & $1.77 \pm 0.02$ & $1.90 \pm 0.04$ & 0.015 \\
\hline TbTh, mm & $0.073 \pm 0.001$ & $0.075 \pm 0.002$ & 0.32 \\
\hline TbSp, mm & $0.53 \pm 0.02$ & $0.49 \pm 0.03$ & 0.31 \\
\hline \multicolumn{4}{|l|}{ Tibia } \\
\hline BV/TV & $0.146 \pm 0.002$ & $0.156 \pm 0.004$ & 0.041 \\
\hline $\mathrm{TbN}, 1 / \mathrm{mm}$ & $1.85 \pm 0.03$ & $2.01 \pm 0.05$ & 0.003 \\
\hline TbTh, mm & $0.080 \pm 0.001$ & $0.079 \pm 0.002$ & 0.68 \\
\hline TbSp, mm & $0.49 \pm 0.01$ & $0.44 \pm 0.02$ & 0.006 \\
\hline \multicolumn{4}{|l|}{ HR-pQCT, cortical } \\
\hline \multicolumn{4}{|l|}{ Radius } \\
\hline Ct vBMD, mg/cm³ & $864 \pm 4.7$ & $880 \pm 8.2$ & 0.11 \\
\hline CtTh, mm & $1.06 \pm 0.02$ & $1.11 \pm 0.03$ & 0.19 \\
\hline Ct porosity & $0.023 \pm 0.001$ & $0.023 \pm 0.001$ & 0.79 \\
\hline \multicolumn{4}{|l|}{ Tibia } \\
\hline Cortical vBMD, $\mathrm{mg} / \mathrm{cm}^{3}$ & $802 \pm 4.6$ & $819 \pm 8.1$ & 0.09 \\
\hline CtTh, mm & $1.24 \pm 0.02$ & $1.29 \pm 0.03$ & 0.27 \\
\hline Cortical porosity & $0.069 \pm 0.002$ & $0.068 \pm 0.003$ & 0.87 \\
\hline
\end{tabular}

Body size and HR-pQCT values were age- and sex-adjusted least squares means \pm SEM. $P$ values are based on linear regression models that include the variables $\beta_{1}$-AR-selective blocker use status, age, and sex. ${ }^{A}$ Users were significantly older $(P<0.001)$ and more likely to be male $(P<0.05)$ than were nonusers. Summary statistics for age represent the mean $\pm \mathrm{SD}$. Ct, cortical; TbSp, trabecular separation; vBMD, volumetric BMD.

es in PINP, but there was otherwise no consistent pattern of baseline catecholamine values predictive of changes in either bone turnover or BMD. We should note that a caveat to these measurements is that sympathetic outflow can be different across tissues, so single plasma measurements should be interpreted with caution.

\section{Discussion}

Using 3 different approaches, we demonstrate in the present study that (a) $\beta_{1}$ - and $\beta_{2}$-ARs, but not $\beta_{3}$-ARs, are expressed in human bone; (b) patients receiving $\beta_{1}$-selective blockers have better bone microarchitecture than do those not treated with $\beta$-blockers; and (c) $\beta_{1}$-selective blockers (atenolol, nebivolol), but not a nonselective $\beta$-AR blocker (propranolol), have favorable effects on bone turnover and BMD in postmenopausal women. Although some correlative evidence, summarized below, has been supportive of a role for SNS regulation of bone metabolism in humans, to our knowledge, this is the first study to establish causality and identify the role of $\beta_{1}$-AR modulation in regulating bone turnover and mass in humans.

These findings are consistent with those of a previous study from our group (21), in which we related sympathetic activity (measured using microneurography at the peroneal nerve) to bone microstructure (assessed by HR-pQCT) in preand postmenopausal women. As with earlier findings (22), we demonstrated in that study that sympathetic outflow was approximately 2.4-fold higher in the postmenopausal women than in the premenopausal women. In the 2 groups combined and after age adjustment, we found that sympathetic activity was inversely correlated with BV/ TV and trabecular thickness (TbTh) at the distal

osteocalcin with both doses of propranolol (Supplemental Figure 5). Collectively, these data demonstrate that atenolol and nebivolol, but not propranolol, consistently reduce bone resorption; although serum PINP was reduced with both $\beta_{1}$-selective blockers, we detected little or no changes in osteocalcin with atenolol or nebivolol treatment.

Figure 4 shows the changes over 20 weeks in the DXA BMD values. Despite the relatively short duration of the intervention, ultradistal radius BMD (which predominantly contains trabecular bone) increased significantly following treatment with atenolol (3.6\% relative to placebo) and nebivolol (2.9\% relative to placebo) (Figure 4A). By contrast, changes at the distal radius (which predominantly contains cortical bone; Figure 4B) were not statistically significant. The overall analysis of covariance (ANCOVA) model (see Statistics) was not significant for changes in femur neck or spine BMD for any of the interventions (Figure 4, C and D).

As the 2 effective drugs were atenolol and nebivolol, we also explored whether baseline plasma catecholamine levels predicted a response to either drug. As shown in Table 4, in the nebivolol group, baseline epinephrine levels were negatively correlated with chang- radius. Similar findings were recently reported by Lamberts et al. (23), who also used microneurography to measure sympathetic outflow in 96 overweight or obese men and women and demonstrated inverse associations between sympathetic outflow and total body and leg bone mineral content.

Our population data showing better bone microarchitecture by HR-pQCT in subjects treated with $\beta_{1}$-selective blockers as compared with nonusers are largely consistent with data from previous observational studies on the relationship of $\beta$-blocker use to BMD and fracture risk. Use of $\beta$-blockers was associated with a $23 \%$ reduction in fracture risk in the UK General Practice Research Database (24), and several recent meta-analyses consistently found protective effects of $\beta$-blocker use on BMD and fracture risk (25-28). The largest of these meta-analyses by Toulis et al. (28) pooled results of 16 studies involving 1,644,570 subjects and found that the risk of any fracture was significantly reduced in subjects receiving $\beta$-blockers as compared with the risk for control subjects (random effects pooled effect size of 0.86; 95\% CI, 0.78$0.93)$, with similar protection seen in women and men. Interestingly, and consistent with our findings, the analysis of Toulis et al. 


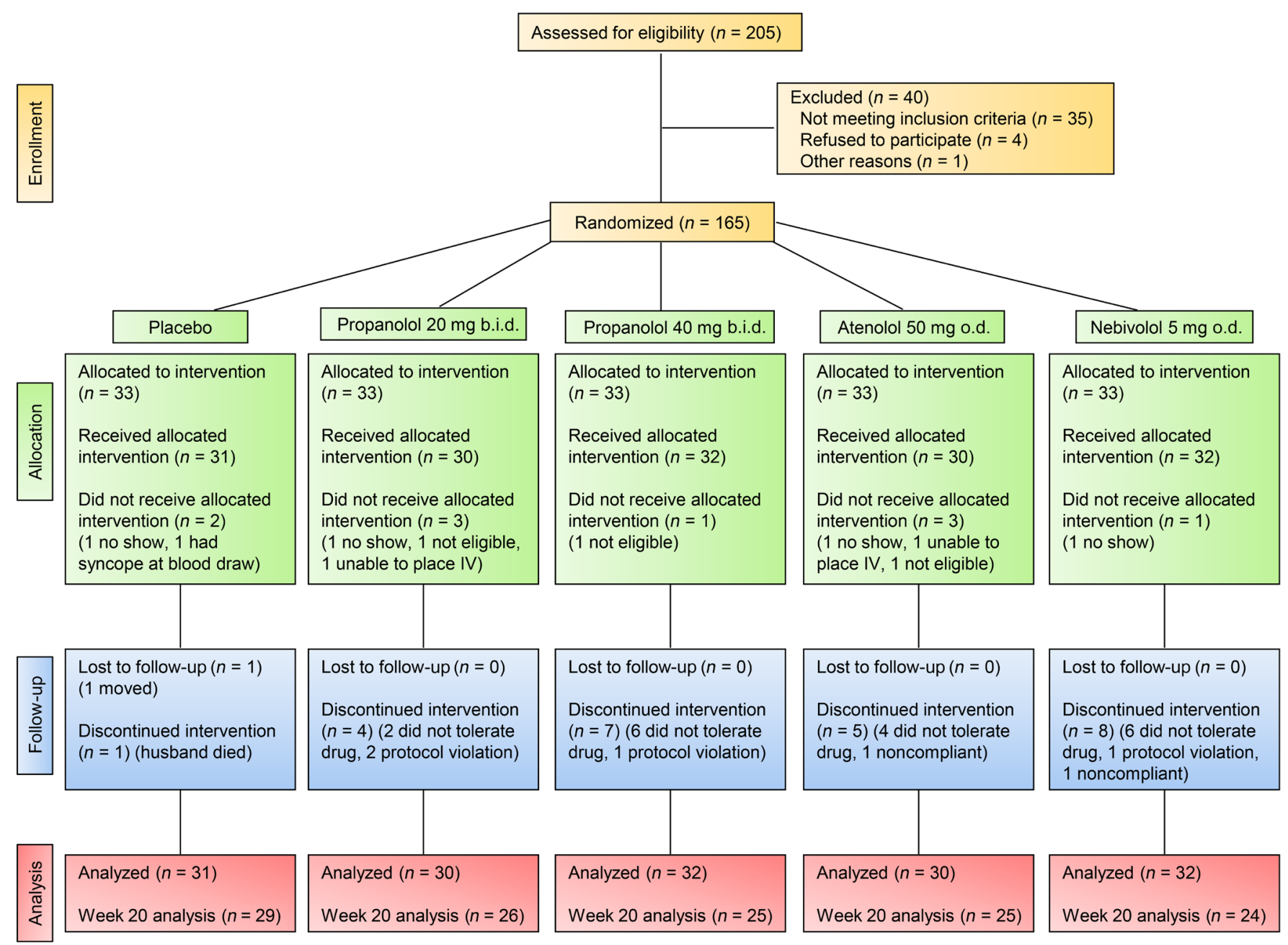

Figure 2. CONSORT flow diagram for the interventional study. The study flow diagram shows the number of subjects assessed for eligibility as well as the number randomized, allocated, lost to follow-up, and included in the final analysis.

(28) as well as an earlier meta-analysis by Yang et al. (27) demonstrated that it was predominantly $\beta_{1}$-selective blockers that were associated with higher BMD and reduced fracture risk.

Studies of patients with pheochromocytomas are also consistent with the hypothesis that excessive catecholamine production by these tumors leading to activation of $\beta$-ARs regulates bone metabolism. Thus, Veldhuis-Vlug et al. (29) examined changes in bone turnover markers following adrenalectomy in 21 patients with pheochromocytomas. Despite the confounding factor that 14 of the 21 patients were on $\beta$-blocker treatment preoperatively, pheochromocytoma resection was associated with a $21 \%$ decrease in serum CTx levels. Consistent with this, Kim et al. (30) found that subjects with pheochromocytomas had 7.2\% lower BMD at the spine as well as 33.5\% higher CTx levels compared with matched subjects without pheochromocytomas. Interestingly, despite the much greater catecholamine production in patients with pheochromocytomas compared with that of normal subjects, the observed decreases in CTx following tumor removal are in the same range that we observed $(20 \%)$ with atenolol or nebivolol treatment.

As noted earlier, a previous study by Reid and colleagues in postmenopausal women treated with $160 \mathrm{mg} /$ day propranolol concluded that $\beta$-blockers did not have favorable effects on bone metabolism in humans (9). In our study, given the animal data showing that lower doses of propranolol had greater skeletal efficacy than did higher doses $(7,8)$, we used $50 \%$ and $25 \%$ of the previously ineffective $160-\mathrm{mg} /$ day propranolol dose (9). Despite this, our findings with propranolol were generally consistent with those of Reid et al. (9), and a similar lack of effects of propranolol at a daily dose of $80 \mathrm{mg} /$ day on serum CTx or PINP levels in postmenopausal women was recently reported by Veldhuis-Vlug et al. (31). Of note, we found that propranolol markedly decreased serum osteocalcin levels; in fact, Reid et al. (9) reported identical changes in serum osteocalcin following propranolol treatment in their study. Whether this reflects a detrimental effect of propranolol on bone metabolism and potentially on bone mass with longer-term treatment remains unclear. Collectively, however, ours and the previous findings provide strong evidence that, unlike findings in rodents $(2,7,8)$, nonselective $\beta$-AR blockade does not have favorable effects on bone metabolism in humans. By contrast, our data demonstrate that in humans, $\beta_{1}$-selective blockers (atenolol and nebivolol) reduce bone resorption and have favorable effects on BMD, at least at the ultradistal radius, 
Table 3. Baseline anthropometric, biochemical, and DXA parameters of study subjects

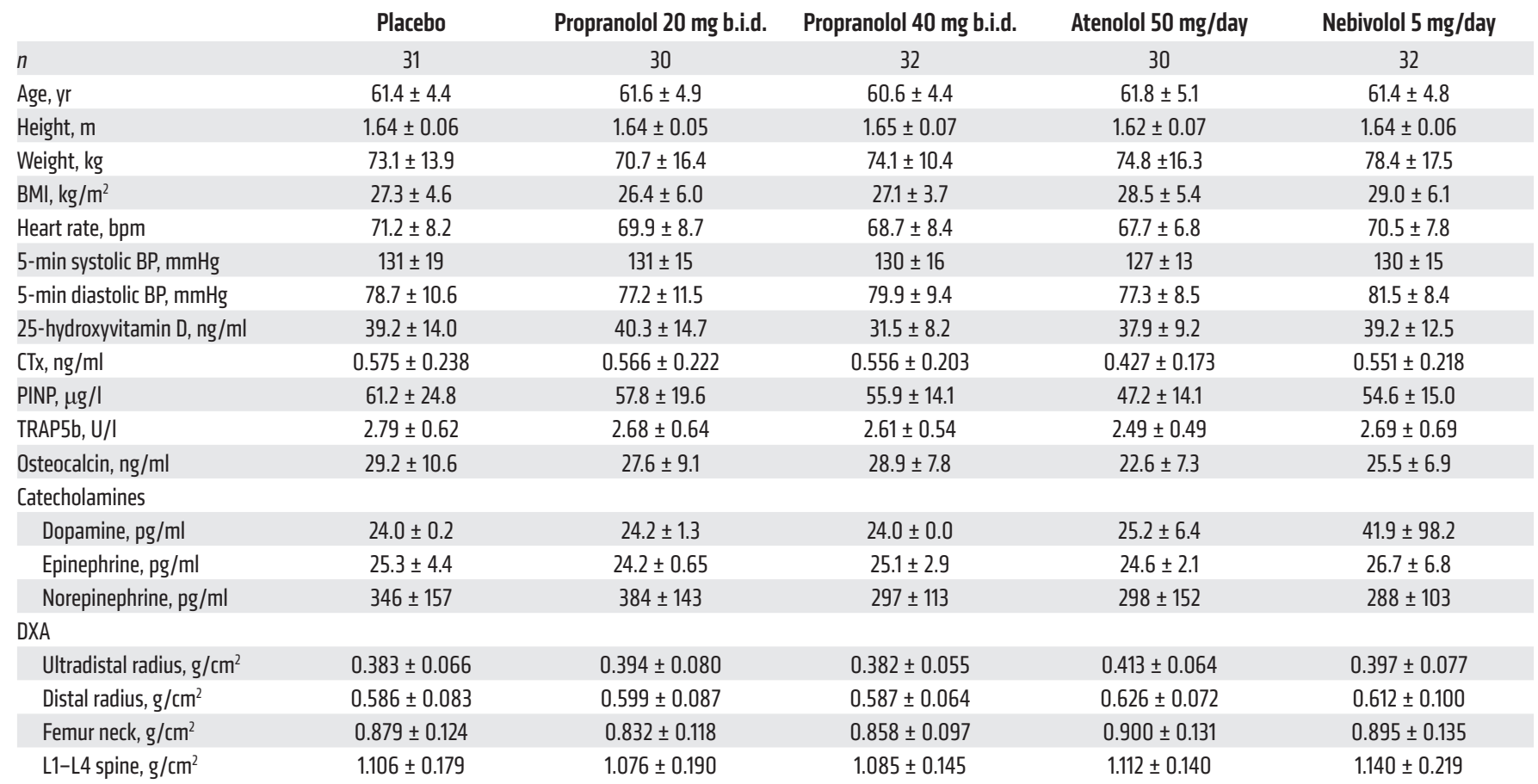

Data represent the mean $\pm \mathrm{SD}$.

a site enriched for trabecular bone, during a treatment period of as brief as 20 weeks.

As noted earlier, available animal data have found that sympathetic outflow increases bone resorption $(3,7,8)$ and decreases bone formation (2). In our interventional study, $\beta_{1}$-AR blockade with atenolol or nebivolol resulted in reductions in markers of bone resorption (CTx, TRAP5b) as well as in serum PINP levels, but had minimal (nebivolol) or no (atenolol) effects on serum osteocalcin levels. In fact, we observed a transient increase (at 2 weeks) in serum PINP levels with nebivolol treatment. Given the previous rodent data demonstrating effects of sympathetic outflow on bone formation (2), it is possible that both atenolol and nebivolol have favorable effects on bone formation but that this effect is masked over time because of the noted reduction in bone resorption leading to a reduction in the coupled process of bone formation, as seen with all antiresorptive agents (32). A favorable effect on bone formation may potentially explain why, in the setting of modest reductions in serum CTx levels of approximately $20 \%$, we observed substantial increases in ultradistal radius BMD with both atenolol and nebivolol over just 20 weeks of treatment.

We acknowledge that the $\beta_{1}$-selective blockers that are clinically available and used in our study are not entirely specific for the $\beta_{1}-\mathrm{AR}$, and it is possible that the observed effects with atenolol and nebivolol may not be due solely to $\beta_{1}$-AR blockade but also to a "low-dose spillover" effect on the $\beta_{2}$-AR. However, if this were the case, we should have observed clearly better skeletal effects with atenolol compared with nebivolol, as the latter is highly specific for $\beta_{1}$-AR blockade, with little or no effects on $\beta_{2}$-ARs $(19,20)$; nonetheless, our data do not formally exclude this possibility. In addition, our data do not establish whether the effects of $\beta_{1}$-AR blockade we observed were due to direct effects on bone or were mediated indirectly, perhaps via CNS $\beta_{1}$-AR blockade. However, although nebivolol is relatively lipophilic and does cross the blood-brain barrier (33), atenolol is not lipophilic and has very low CNS penetration (34). Accordingly, the relatively similar effects of both drugs on bone turnover and BMD would argue against central $\beta_{1}$-AR blockade as a major factor in our findings. Nonetheless, it is still possible that the effects of $\beta_{1}$-AR-selective blockers on bone were mediated indirectly, for example, through reductions in peripheral vascular resistance and increased skeletal blood flow or via other hormonal changes (e.g., in growth hormone or IGF 1 levels) that we did not measure. Finally, we also did not specifically evaluate changes in physical activity or mental/cognitive function following each of our interventions that could lead to changes in the biomechanical impact on the skeleton, so we cannot exclude the possibility that these factors may have played a role in our findings.

Since propranolol also binds the $\beta_{1}-\mathrm{AR}$, it is perhaps surprising that it lacked efficacy in regulating bone metabolism in humans. However, the relative affinity ratios $\left(\beta_{1} / \beta_{2}\right)$ for the 3 drugs used in this study were propranolol: 0.3 ; atenolol: 5.7 ; and nebivolol: 40.0 (20). As such, if the skeletal response depends specifically on $\beta_{1}$ AR antagonism or the ratio of $\beta_{1}$ versus $\beta_{2}$ affinity, that may explain our findings. It is also of interest that in hFOB cells, both a $\beta_{1}-\mathrm{AR}$ agonist (dobutamine) $(12,13)$ and a $\beta_{2}$-AR agonist (salmeterol) (14) increased RANKL mRNA levels, and both agonists increased $A D R B 1$ but not ADRB2 mRNA levels. These findings suggest the possibility that the higher sympathetic outflow that we (21) and others (22) have demonstrated in postmenopausal versus pre- 

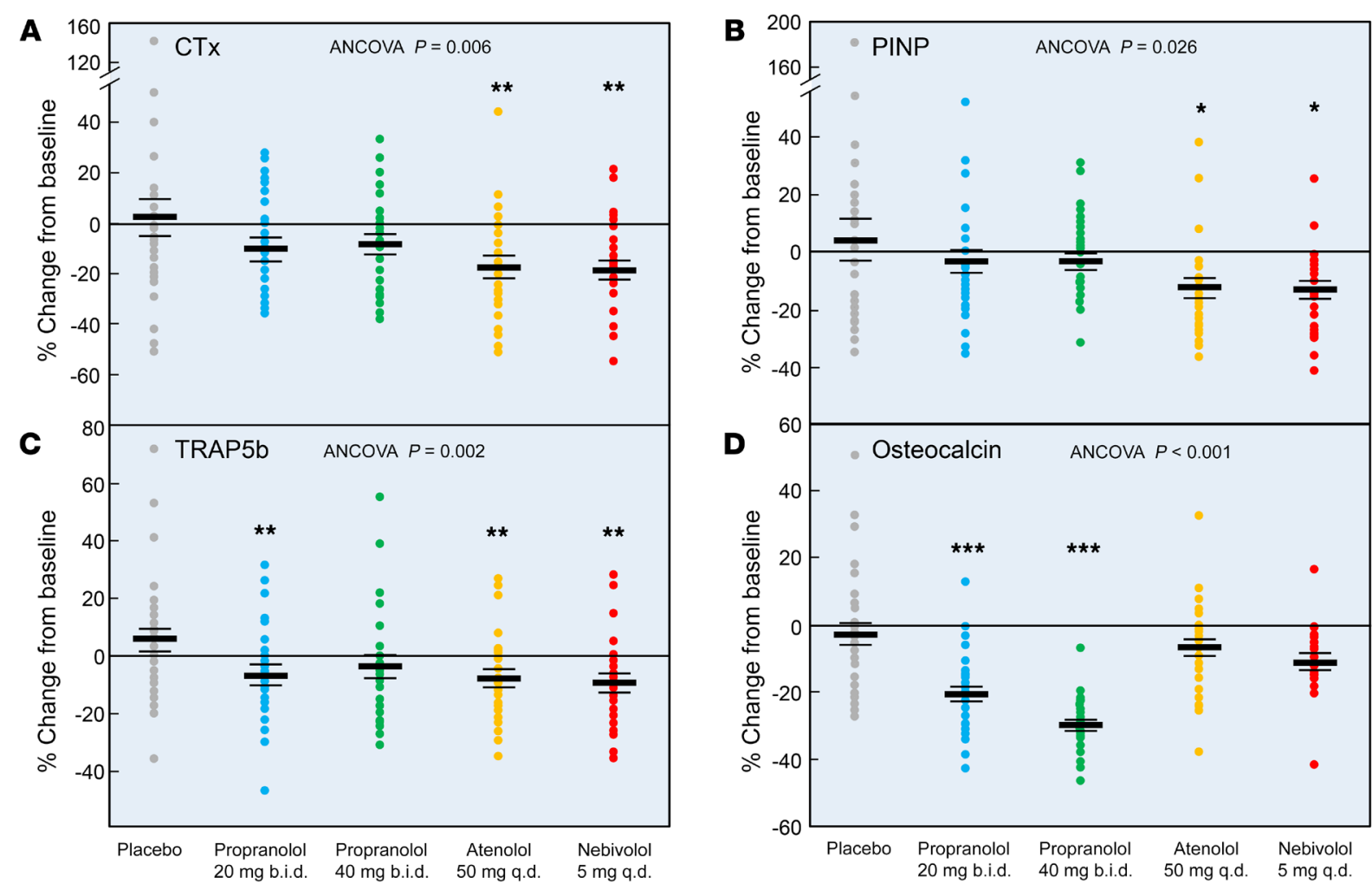

Figure 3. Percentage of change from baseline in bone resorption and bone formation markers in the study subjects following the assigned treatments. Percentage of change from baseline after 20 weeks of treatment in serum levels of (A) CTx, (B) PINP, (C) TRAP5b, and (D) osteocalcin. ANCOVA P values are indicated, and when these were less than 0.05 , individual groups were compared with the placebo-treated group using the Dunnett's 2-tailed $t$ test.

${ }^{*} P<0.05,{ }^{* *} P<0.01$, and ${ }^{* *} P<0.001$. As per the CONSORT flow diagram in Figure 2, the sample sizes for the week-20 analyses were as follows: placebo, $n=29$; propranolol $20 \mathrm{mg}$ b.i.d., $n=26$; propranolol $40 \mathrm{mg}$ b.i.d., $n=25$; atenolol $50 \mathrm{mg} /$ day, $n=25$; and nebivolol $5 \mathrm{mg} /$ day, $n=24$.

menopausal women may induce a "feed-forward" loop in bone by increasing the expression of $A D R B 1$, thereby increasing the relative importance of signaling through this receptor with aging and perhaps explaining why the $\beta_{1}$-selective antagonists were efficacious in reducing bone resorption in our study but a nonselective $\beta$-antagonist was not. Arguing against this explanation is the fact that we did not find an increase in ADRB1 mRNA in bone biopsies from postmenopausal women as compared with those from premenopausal women, although it is possible that $A D R B 1$ expression increased in a specific population of osteoblastic cells in the bone biopsies but was masked by the heterogeneous population of cells in the biopsies. Further studies are clearly needed to better define the underlying mechanisms explaining the better skeletal efficacy of $\beta_{1}$-selective antagonists as compared with a nonselective $\beta$-antagonist, although the differential regulation of $A D R B 1$ versus $A D R B 2$ by $\beta$-adrenergic agonists in human osteoblastic cells may provide a clue regarding potential directions to pursue.

In conclusion, using 3 independent approaches, we believe our study establishes for the first time an important role for adrenergic signaling in the regulation of bone metabolism in humans, principally via $\beta_{1}$-ARs. Given the previously noted increase in sympathetic outflow in postmenopausal versus premenopausal women (21) and with aging in both sexes (22), our findings raise the testable hypothesis that increased $\beta$-adrenergic signaling may contribute to postmenopausal and age-related bone loss. In addition, our study also has potential therapeutic implications. Thus, although a number of options exist for the treatment of osteoporosis, concerns regarding rare side effects such as osteonecrosis of the jaw or atypical femur fractures have led to a marked reduction in the appropriate use of the available drugs (35). Moreover, following dissemination of the results of the Women's Health Initiative (36), a substantial number of women who were previously treated with estrogen for the prevention of osteoporosis now have few, if any, options, because bisphosphonates are now generally only recommended for women already at high risk of fracture (37). As such, the possibility that relatively safe and inexpensive (at least for generic atenolol) drugs such as $\beta_{1}$-AR-selective blockers that have been widely used in the treatment of hypertension, migraine, congestive heart failure, and other cardiovascular diseases (38) may also have utility for the prevention or treatment of osteoporosis warrants further investigation. Specifically, our findings establishing proof of concept that $\beta_{1}$-AR-selective blockers may have favorable effects on bone turnover and BMD in postmenopausal women provide compelling data to support the initiation of longer-term, definitive clinical trials with BMD and/or fracture as outcomes to evaluate whether these agents can fill this crucial clinical need.

\section{Methods}

\section{Study subjects}

Biopsy study. We reanalyzed needle bone biopsies from a previous study (10) involving 19 young women (mean age \pm SD $30.3 \pm 5.4$ years) 

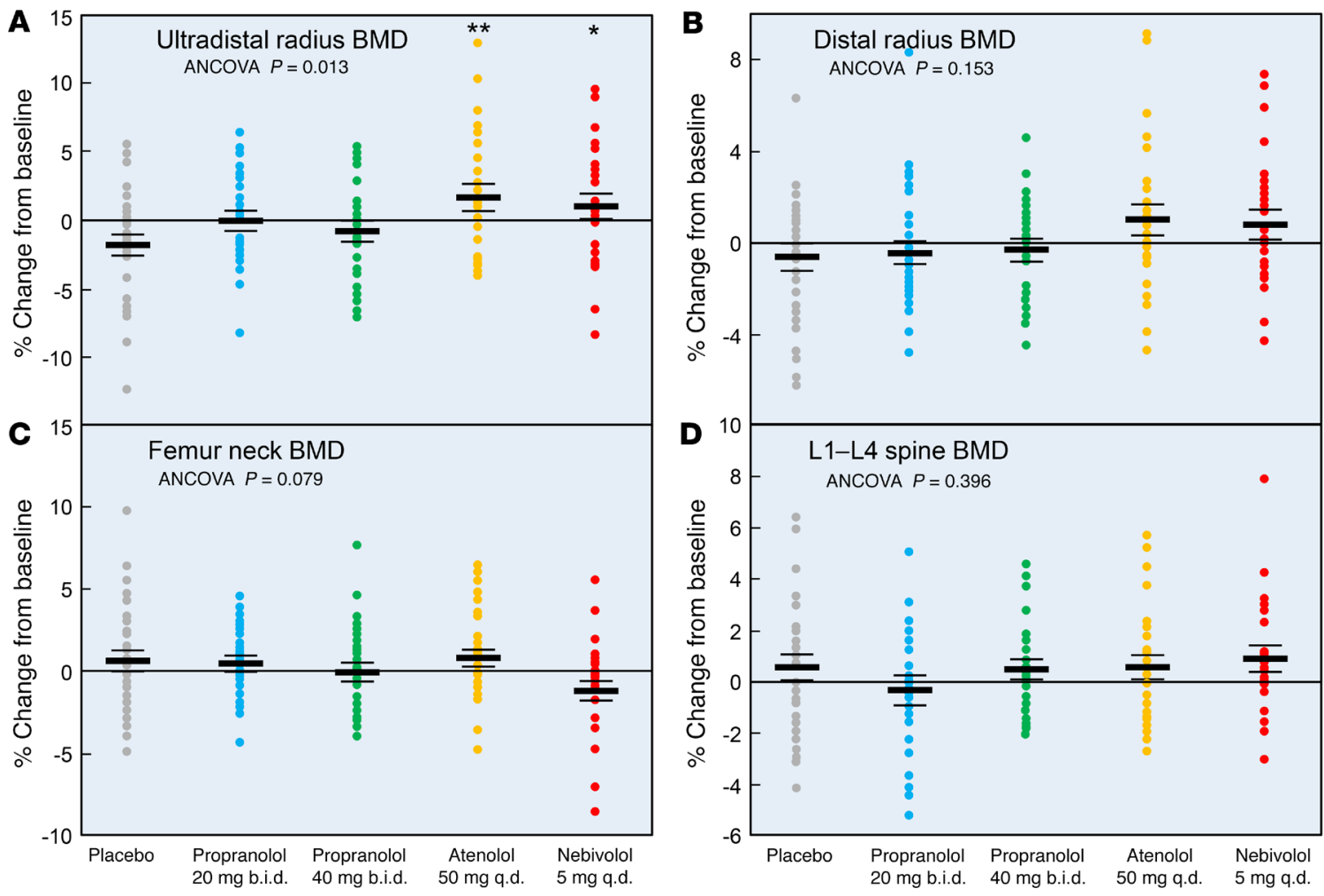

Figure 4. Percentage of change from baseline in BMD in the study subjects following the assigned treatments. Percentage of $B M D$ change from baseline following 20 weeks of treatment at the (A) ultradistal radius, (B) distal radius, (C) femur neck, and (D) lumbar spine. ANCOVA $P$ values are indicated, and when these were less than 0.05 , individual groups were compared with the placebo-treated group using a Dunnett's 2 -tailed $t$ test. ${ }^{*} P<0.05$ and ${ }^{* *} P<$ 0.01. As per the CONSORT flow diagram in Figure 2, the sample sizes for the week-20 analyses were as follows: placebo, $n=29$; propranolol $20 \mathrm{mg}$ b.i.d., $n=26$; propranolol $40 \mathrm{mg}$ b.i.d., $n=25$; atenolol $50 \mathrm{mg} /$ day, $n=25$; and nebivolol $5 \mathrm{mg} /$ day, $n=24$.

and 19 elderly women (73.1 \pm 6.6 years). Details regarding the inclusion and exclusion criteria for these subjects and the procedures for RNA-seq are provided in our previous publication (10).

Cell culture studies. The human osteoblast cell line hFOB was obtained from the laboratory of Thomas Spelsberg (Mayo Clinic, Rochester, Minnesota, USA), which originally established this line (11). hFOB cells were plated at $2.5 \times 10^{4}$ cells $/ \mathrm{cm}^{2}$ in 12 -well plates and cultured in aMEM supplemented with $1 \times$ antibiotic/antimycotic (Thermo Fisher Scientific), 1× Glutamax ,and 10\% (v/v) FBS (HyClone Laboratories, GE Healthcare Life Sciences). Following standard culture conditions for 72 hours, the cells were treated with either vehicle, $25 \mu \mathrm{M}$ dobutamine, or $10 \mathrm{nM}$ salmeterol for 2 hours. The doses were based on detailed dose response studies by Yin et al. (39) for dobutamine and Baker (40) for salmeterol; both reagents were obtained from Sigma-Aldrich. RNA isolation, cDNA synthesis, and quantitative reverse transcriptase PCR (qPCR) using ACTB and TUBA1A as stable references genes were performed as previously described (41). The primer sequences for the genes analyzed are provided in Supplemental Table 2.

Population cohort. Details regarding the population cohort included in this study have been provided previously $(17,18)$. Subjects were recruited from an age-stratified random sample of Rochester, Minnesota, residents who were selected using the medical records linkage system of the Rochester Epidemiology Project (42). This population is highly characteristic of the US White population, but Blacks and Asians are underrepresented (42). Specific details regarding this cohort, including the HR-pQCT imaging findings, have previously been described $(17,18)$. Briefly, the cohort study included all subjects aged 50 years and older from our previously described populationbased sample $(17,18)$, all of whom had HR-pQCT measurements and were not undergoing treatment with any medications that affect bone turnover (e.g., estrogen therapy, bisphosphonates, selective estrogen receptor modulators). Four subjects who were on a nonselective $\beta$-blocker (propranolol) in the previous 5 years were excluded. The remaining subjects were divided into those who had used $\beta_{1}$-AR-selective blockers (atenolol or metoprolol) in the previous 5 years $(n=63)$; all remaining subjects $(n=185)$ were considered nonusers. As this was a random sample from the population, there were no obvious differences in risk factors for low BMD between $\beta$-blocker users and nonusers. For example, there were no statistically significant differences between the 2 groups for recent corticosteroid use ( $11 \%$ of $\beta$-blocker users vs. $15 \%$ of nonusers; unadjusted $P=0.43$; age- and sex-adjusted $P=0.34$ ) or corticosteroid use ever (35\% of $\beta$-blocker users vs. $43 \%$ of nonusers; unadjusted $P=0.25$; age- and sex-adjusted $P=0.22$ ).

Interventional study. As shown in the CONSORT diagram (Figure 2) for the study (ClinicalTrials.gov NCT02467400), we recruited healthy postmenopausal women (follicle-stimulating hormone [FSH] $>20 \mathrm{IU} / \mathrm{l}$ ) aged 50-70 years who were in good health. The exclusion criteria were as follows: (a) abnormality in any of the screening laboratory studies (complete blood count, serum calcium, phosphorus, creatinine, and 25-hydroxyvitamin D levels); (b) presence of significant liver or renal disease, malignancy (including myeloma), malabsorption, diabetes, hypoparathyroidism, hyperparathyroidism, acromegaly, Cushing's 
Table 4. Spearman's correlation coefficients for the relationship of baseline catecholamine levels to the percentage changes in bone turnover and DXA parameters in response to atenolol and nebivolol

\begin{tabular}{|c|c|c|c|c|c|c|c|c|}
\hline & CTx & PINP & TRAP5b & Osteocalcin & $\begin{array}{c}\text { Ultradistal radius } \\
\text { BMD }\end{array}$ & $\begin{array}{l}\text { Distal radius } \\
\text { BMD }\end{array}$ & $\begin{array}{c}\text { Femur neck } \\
\text { BMD }\end{array}$ & $\begin{array}{l}\text { Spine } \\
\text { BMD }\end{array}$ \\
\hline \multicolumn{9}{|l|}{ Atenolol } \\
\hline \multicolumn{9}{|l|}{ Catecholamines } \\
\hline Dopamine & 0.26 & -0.02 & 0.18 & 0.23 & -0.03 & 0.03 & 0.22 & -0.18 \\
\hline Epinephrine & 0.13 & -0.03 & 0.15 & 0.15 & 0.03 & 0.06 & -0.21 & -0.02 \\
\hline Norepinephrine & -0.02 & 0.02 & -0.35 & -0.08 & -0.28 & -0.24 & -0.28 & -0.08 \\
\hline \multicolumn{9}{|l|}{ Nebivolol } \\
\hline \multicolumn{9}{|l|}{ Catecholamines } \\
\hline Dopamine & 0.37 & 0.06 & -0.16 & 0.10 & 0.05 & -0.02 & -0.30 & 0.06 \\
\hline Epinephrine & -0.02 & $-0.46^{A}$ & -0.23 & -0.31 & -0.02 & -0.09 & -0.08 & -0.12 \\
\hline Norepinephrine & 0.39 & 0.01 & 0.04 & -0.12 & 0.08 & 0.05 & 0.17 & -0.20 \\
\hline${ }^{A} P<0.05$ & & & & & & & & \\
\hline
\end{tabular}

nebivolol) and large volume of tablets, we did not have the resources to cover the cost of manually encapsulating each tablet at our research pharmacy to make all tablets indistinguishable. Study staff were blinded, because a research pharmacist not involved in the study provided each subject with 1 bottle labeled "am" and a second bottle labeled "pm," with 1 tablet to be taken from each bottle in the morning and evening, respectively. As such, study staff never saw the actual tablets any subject was taking. None of

syndrome, hypopituitarism, or severe chronic obstructive pulmonary disease; (c) undergoing treatment with any medications that affect bone turnover, including the following: adrenocorticosteroids ( $>3$ months at any time or $>10$ days within the previous year); anticonvulsant therapy (within the previous year); pharmacological doses of thyroid hormone (causing a decline of thyroid-stimulating hormone below normal levels); calcium supplementation of $>1,200 \mathrm{mg} /$ day (within the preceding 3 months); bisphosphonates (within the past 3 years); denosumab; estrogen therapy or treatment with a selective estrogen receptor modulator; or teriparatide (within the previous year). Subjects with a clinical history of osteoporotic fracture (vertebral, hip, or distal forearm) were also excluded, as were subjects with any recent (within the previous 6 months) fracture. For vitamin D sufficiency, we ensured that all subjects had serum 25-hydroxyvitamin D levels of greater than $20 \mathrm{ng} / \mathrm{ml}$. If, at the screening visit, a subject was found to have low body stores of vitamin $\mathrm{D}$ as assessed by serum 25-hydroxyvitamin D levels below $20 \mathrm{ng} / \mathrm{ml}$ ( $n=14$ subjects), the subject was treated with $1,000 \mathrm{U} /$ day vitamin D for 8 weeks and then had this level rechecked; all subjects achieved serum 25-hydroxyvitamin D levels of greater than $20 \mathrm{ng} / \mathrm{ml}$ and then proceeded with the study. Additional exclusion criteria included a resting blood pressure (BP) above 150/90 $\mathrm{mmHg}$ or hypotension (systolic $\mathrm{BP}<100 \mathrm{mmHg}$ ), a heart rate below $60 \mathrm{bpm}$ at the screening visit (the average of 3 heart rate and BP measurements, separated by at least 1 minute), use of antihypertensive medication, or a history of asthma.

\section{Procedures and treatment}

Study protocols. The protocols for the biopsy component (10) and population sample $(17,18)$ have been described previously. For the interventional study, the subjects were randomly assigned to 1 of 5 treatment groups: placebo (b.i.d.); propranolol $40 \mathrm{mg}$ /day (20 mg b.i.d.); propranolol $80 \mathrm{mg} /$ day (40 mg b.i.d.); atenolol, $50 \mathrm{mg} /$ day; or nebivolol, $5 \mathrm{mg} /$ day (subjects in the once-daily groups received a placebo as the second dose so that all subjects took 2 pills per day). Block randomization was used so that equal numbers of subjects were assigned to each treatment arm for each block of 15 subjects. The interventional trial was a randomized, placebo-controlled study with random allocation. Given the number of drugs (placebo, propranolol, atenolol, and the tablets had drug identifiers, but because not all the tablets looked identical, it is possible that some subjects searched the internet and were able to discern which arm they were randomized to. Although we requested that study subjects remain blinded to the drug, we cannot exclude the possibility that this was not respected. Nonetheless, as our endpoints were all objective measures, we believe that this had little, if any, impact on the study. Throughout the study, all participants took the 800 IU vitamin D that was supplied to them, unless they were already taking a multivitamin that contained at least 500 IU vitamin D. These subjects were instructed to continue taking their multivitamin daily throughout the study. Following admission to the Mayo Outpatient Clinical Research and Trials Unit (CRTU), the subjects rested for 5 minutes, following which the baseline resting heart rate and BP were measured. Fasting blood samples were obtained for baseline assessment of bone formation (serum PINP and OCN) and resorption markers (serum CTx and TRAP5b), as well as for additional assays. Assays were performed in serum or plasma that had been stored at $-80^{\circ} \mathrm{C}$. At the baseline visit, a plasma sample was also obtained for the measurement of catecholamines (epinephrine, norepinephrine, and dopamine) using a standardized protocol (patient resting in the supine position in a quiet room for 30 minutes and blood drawn through an indwelling catheter). Study subjects returned as outpatients to the CRTU for fasting morning blood samples at weeks 1, 2, 4, 8, 12, 16, and 20 (window of \pm 2 days for each of these visits). All of the parameters measured at baseline were measured at each of these time points, except for the plasma catecholamines, which were only measured at baseline. Ultradistal radius, distal radius, L1-L4 spine, and femur neck BMD by DXA (Lunar iDXA, GE Medical Systems using enCORE software, version 15.0) were measured at baseline and 20 weeks.

Biochemical assays. Serum CTx was measured using the Onestep ELISA Kit (Nordic Bioscience Diagnostics, coefficient of variation $[\mathrm{CV}]<8 \%$ ); PINP was measured by RIA (Orion Diagnostica, CV $<9 \%$ ); TRAP5b was measured by ELISA (Immunodiagnostic Systems Ltd., CV <14\%); and osteocalcin was measured using the ELSAOsteo Two-site immunoradiometric assay (IRMA) (Cisbio US, CV $<8 \%$ ). Levels of 25-hydroxyvitamin D [25(OH)D] (CV <7\%) were measured using liquid chromatography-tandem mass spectrometry (API 5000; Applied Biosystems/MDS SCIEX). Plasma catechol- 
amines (dopamine, epinephrine, norepinephrine) were measured using HPLC in the Mayo Clinical Laboratories (CV $<13 \%)$.

\section{Statistics}

Details regarding processing and analysis of the RNA-seq data have been previously published (10). For analysis of the population cohort, values are presented as age- and sex-adjusted least squares means, and linear regression models were used to assess each parameter including the variables age, sex, and $\beta_{1}$-selective blocker use status. Spearman's correlations were used to summarize the relationship between baseline catecholamine levels and changes in bone turnover and DXA parameters. For analysis of the 20-week measurements, an ANCOVA model was used that included treatment as a fixed effect and baseline measurements as a covariate. Although randomization should ideally obviate the need to correct for baseline factors, given that we had 5 groups and approximately 30 subjects per group, there is the possibility that randomization may not have perfectly balanced the subjects with regard to potential covariates - specifically, the baseline values of the respective response variables. A detailed analysis of ANOVA versus ANCOVA by Van Breukelen (43) concluded that for randomized studies with pre- and postintervention measurements, both methods are appropriate and unbiased, but the ANCOVA has more power, and ANCOVA was thus recommended in general. For this reason, we used an ANCOVA model that included the baseline value for each response variable as a covariate in the model. An $F$ test was used to test for overall differences between the treatment groups. When the ANCOVA $P$ value was less than 0.05 , pairwise comparisons between the $\beta$-blocker-treated groups and the placebo-treated group were further assessed using Dunnett's 2-tailed $t$ test, which is a multiple comparisons procedure for comparisons with a control group. For analyses using all the time points, we used a mixed-effects model, allowing the treatment effect to vary across time. This approach allowed for inclu- sion of all subjects including those who did not contribute data at all the time points. All analyses were run using the R Project software package (http://www.R-project.org).

\section{Study approval}

All human studies were approved by the Mayo Clinic IRB, and written informed consent was obtained from all subjects.

\section{Author contributions}

SK, MTD, SJA, EJA, MJJ, CJR, DGM, and JNF participated in the design of the study. TLV recruited the study participants and executed the study, which was overseen by SK, MTD, SJA, EJA, and JNF. BST was responsible for all sample processing and some of the laboratory assays, as well as submitting other laboratory assays to the Mayo Clinic Immunochemical Core Laboratory and Clinical Laboratory. Primary data analysis was performed by SJA and EJA. SK wrote the first draft of the manuscript, which was reviewed, edited, and approved by all authors.

\section{Acknowledgments}

The authors would like to thank the staff of the Mayo CRTU for their help with the studies. We would also like to thank the Mayo Immunochemical Core Laboratory and Clinical Laboratory for performing the assays, as well as James Peterson for help in managing the data and preparing the figures for publication. This research was supported by NIH grants AG004875 and AR027065 and a Mayo Clinic Clinical and Translational Science Award (CTSA) (UL1 TR002377).

Address correspondence to: Sundeep Khosla, Mayo Clinic, Guggenheim 7, 200 First Street SW, Rochester, Minnesota, 55905, USA. Phone: 507.255.6663; Email: khosla.sundeep@mayo.edu.
1. Ducy P, et al. Leptin inhibits bone formation through a hypothalamic relay: a central control of bone mass. Cell. 2000;100(2):197-207.

2. Takeda S, et al. Leptin regulates bone formation via the sympathetic nervous system. Cell. 2002;111(3):305-317.

3. Elefteriou $\mathrm{F}$, et al. Leptin regulation of bone resorption by the sympathetic nervous system and CART. Nature. 2005;434(7032):514-520.

4. Kajimura D, et al. Genetic determination of the cellular basis of the sympathetic regulation of bone mass accrual. J Exp Med. 2011;208(4):841-851.

5. Kellenberger S, Muller K, Richener H, Bilbe G. Formoterol and isoproterenol induce c-fos gene expression in osteoblast-like cells by activating beta2-adrenergic receptors. Bone. 1998;22(5):471-478.

6. Pierroz DD, et al. Deletion of $\beta$-adrenergic receptor 1,2 , or both leads to different bone phenotypes and response to mechanical stimulation. J Bone Miner Res. 2012;27(6):1252-1262.

7. Bonnet N, Laroche N, Vico L, Dolleans E, Benhamou CL, Courteix D. Dose effects of propranolol on cancellous and cortical bone in ovariectomized adult rats. J Pharmacol Exp Ther. 2006;318(3):1118-1127.

8. Sato T, Arai M, Goto S, Togari A. Effects of propranolol on bone metabolism in sponta- neously hypertensive rats. J Pharmacol Exp Ther. 2010;334(1):99-105.

9. Reid IR, et al. Effects of a beta-blocker on bone turnover in normal postmenopausal women: a randomized controlled trial. J Clin Endocrinol Metab. 2005;90(9):5212-5216.

10. Farr JN, et al. Effects of age and estrogen on skeletal gene expression in humans as assessed by RNA sequencing. PLoS One. 2015;10(9):e0138347.

11. Harris SA, Enger RJ, Riggs BL, Spelsberg TC. Development and characterization of a conditionally immortalized human fetal osteoblastic cell line. J Bone Miner Res. 1995;10(2):178-186.

12. Williams RS, Bishop T. Selectivity of dobutamine for adrenergic receptor subtypes: in vitro analysis by radioligand binding. J Clin Invest. 1981;67(6):1703-1711.

13. Tuttle RR, Mills J. Dobutamine: development of new catecholamine to selectively increase cardiac contractility. Circ Res. 1975;36(1):185-196.

14. Sears MR, Lötvall J. Past, present and future-beta2-adrenoceptor agonists in asthma management. Respir Med. 2005;99(2):152-170.

15. Fu L, Patel MS, Karsenty G. The circadian modulation of leptin-controlled bone formation. Prog Brain Res. 2006;153:177-188.

16. Fu L, Patel MS, Bradley A, Wagner EF, Karsenty
G. The molecular clock mediates leptin-regulated bone formation. Cell. 2005;122(5):803-815.

17. Khosla S, et al. Effects of sex and age on bone microstructure at the ultradistal radius: a population-based noninvasive in vivo assessment. J Bone Miner Res. 2006;21(1):124-131.

18. Khosla S, Melton LJ, Achenbach SJ, Oberg AL, Riggs BL. Hormonal and biochemical determinants of trabecular microstructure at the ultradistal radius in women and men. JClin Endocrinol Metab. 2006;91(3):885-891.

19. Nuttall SL, Routledge HC, Kendall MJ. A comparison of the beta1-selectivity of three beta1-selective beta-blockers. JClin Pharm Ther. 2003;28(3):179-186.

20. Ladage D, Schwinger RH, Brixius K. Cardioselective beta-blocker: pharmacological evidence and their influence on exercise capacity. Cardiovasc Ther. 2013;31(2):76-83.

21. Farr JN, et al. Relationship of sympathetic activity to bone microstructure, turnover, and plasma osteopontin levels in women. J Clin Endocrinol Metab. 2012;97(11):4219-4227.

22. Wallin BG, Charkoudian N. Sympathetic neural control of integrated cardiovascular function: insights from measurement of human sympathetic nerve activity. Muscle Nerve. 2007;36(5):595-614. 


\section{CLINICAL MEDICINE}

23. Lambert E, et al. Inverse association between sympathetic nervous system activity and bone mass in middle aged overweight individuals. Bone. 2018;111:123-128.

24. Schlienger RG, Kraenzlin ME, Jick SS, Meier CR. Use of beta-blockers and risk of fractures. JAMA. 2004;292(11):1326-1332.

25. Wiens M, Etminan M, Gill SS, Takkouche B. Effects of antihypertensive drug treatments on fracture outcomes: a meta-analysis of observational studies. JIntern Med. 2006;260(4):350-362.

26. Yang S, Nguyen ND, Eisman JA, Nguyen TV. Association between beta-blockers and fracture risk: a Bayesian meta-analysis. Bone. 2012;51(5):969-974.

27. Yang S, Nguyen ND, Center JR, Eisman JA, Nguyen TV. Association between beta-blocker use and fracture risk: the Dubbo Osteoporosis Epidemiology Study. Bone. 2011;48(3):451-455.

28. Toulis KA, Hemming K, Stergianos S, Nirantharakumar K, Bilezikian JP. $\beta$-Adrenergic receptor antagonists and fracture risk: a meta-analysis of selectivity, gender, and site-specific effects. Osteoporos Int. 2014;25(1):121-129.

29. Veldhuis-Vlug AG, El Mahdiui M, Endert E, Heijboer AC, Fliers E, Bisschop PH. Bone resorption is increased in pheochromocytoma patients and normalizes following adrenalectomy. J Clin Endocrinol Metab. 2012;97(11):E2093-E2097.

30. Kim BJ, et al. Lower bone mass and higher bone resorption in pheochromocytoma: importance of sympathetic activity on human bone. J Clin Endocrinol Metab. 2017;102(8):2711-2718.

31. Veldhuis-Vlug AG, et al. The effects of beta-2 adrenergic agonist and antagonist on human bone metabolism: a randomized controlled trial. Bone. 2015;71:196-200.

32. Khosla S. Odanacatib: location and timing are everything. J Bone Miner Res. 2012;27(3):506-508.

33. Poirier L, Tobe SW. Contemporary use of $\beta$-blockers: clinical relevance of subclassification. Can J Cardiol. 2014;30(5 Suppl):S9-S15.

34. Neil-Dwyer G, Bartlett J, McAinsh J, Cruickshank JM. Beta-adrenoceptor blockers and the blood-brain barrier. Br J Clin Pharmacol. 1981;11(6):549-553.

35. Khosla S, Hofbauer LC. Osteoporosis treatment: recent developments and ongoing challenges. Lancet Diabetes Endocrinol. 2017;5(11):898-907.

36. Rossouw JE, et al. Risks and benefits of estrogen plus progestin in healthy postmenopausal women: principal results From the Women's
Health Initiative randomized controlled trial. JAMA. 2002;288(3):321-333.

37. Khosla S, et al. Benefits and risks of bisphosphonate therapy for osteoporosis. JClin Endocrinol Metab. 2012;97(7):2272-2282.

38. Park CS, Lee HY. Clinical utility of sympathetic blockade in cardiovascular disease management. Expert Rev Cardiovasc Ther. 2017;15(4):277-288.

39. Yin J, et al. Antitumor activity of dobutamine on human osteosarcoma cells. Oncol Lett. 2016;11(6):3676-3680.

40. Baker JG. The selectivity of beta-adrenoceptor agonists at human beta1-, beta2- and beta3-adrenoceptors. Br J Pharmacol. 2010;160(5):1048-1061.

41. Farr JN, et al. Identification of senescent cells in the bone microenvironment. JBone Miner Res. 2016;31(11):1920-1929.

42. Rocca WA, Yawn BP, St Sauver JL, Grossardt BR, Melton LJ. History of the Rochester Epidemiology Project: half a century of medical records linkage in a US population. Mayo Clin Proc. 2012;87(12):1202-1213.

43. Van Breukelen GJ. ANCOVA versus change from baseline: more power in randomized studies, more bias in nonrandomized studies [corrected]. JClin Epidemiol. 2006;59(9):920-925. 